\title{
Acute disseminated encephalomyelitis in children and adolescents
}

Lorna Stemberger Marići ${ }^{1,2}$, Ivan Ledinski ${ }^{1}$, Goran Tešović1,3

Background: Acute disseminated encephalomyelitis (ADEM) is a rare, usually monophasic inflammatory demyelinating disease of central nervous system. Nonspecific clinical manifestations and absence of reliable biochemical markers, makes the diagnosis based on clinical and paraclinical data.

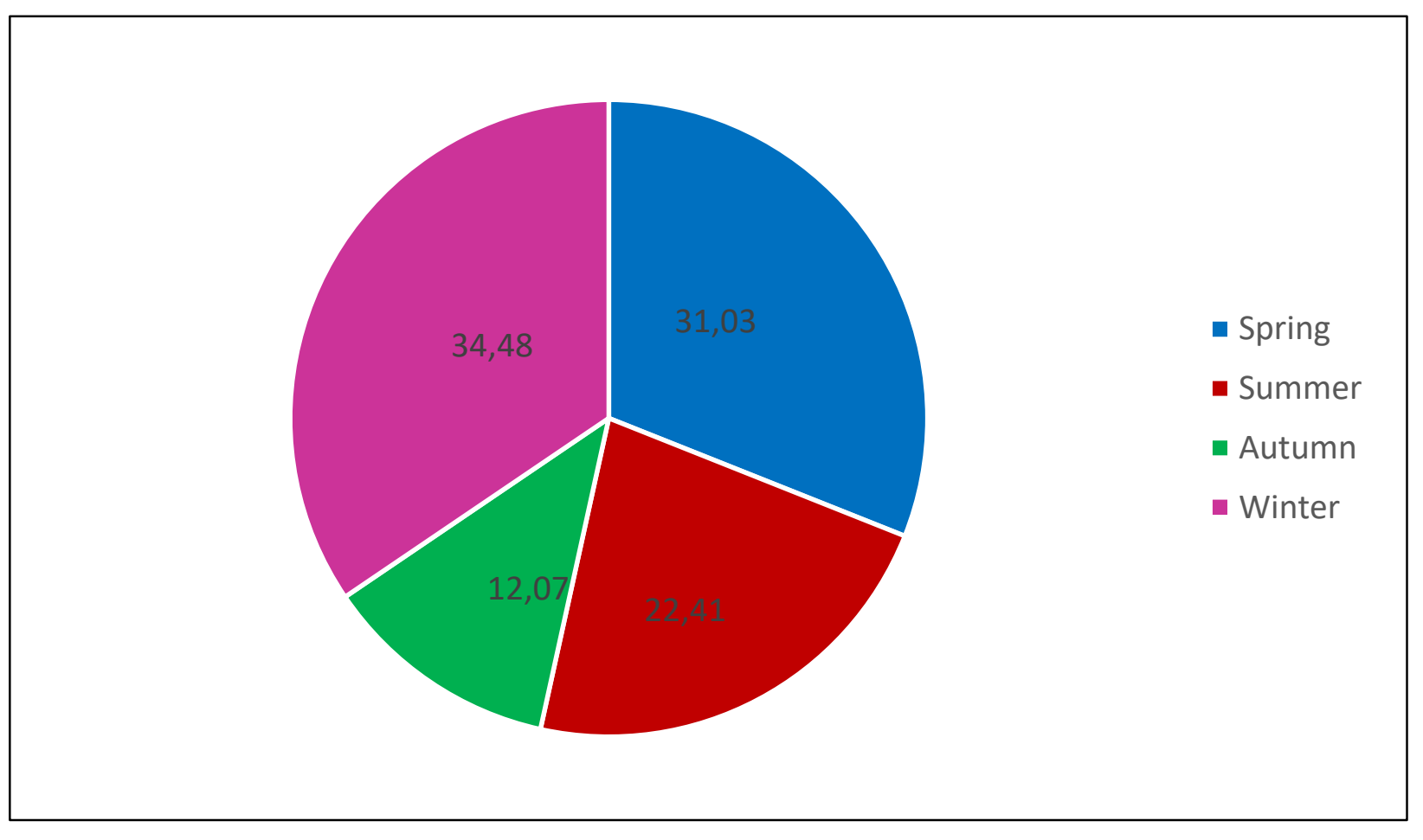

Materials/methods: We conducted retrospective study of 58 pediatric patients with ADEM treated in University hospital for infectious diseases diseases Zagreb in aim to identify distinguishing features of the disease which would facilitate early diagnosis.

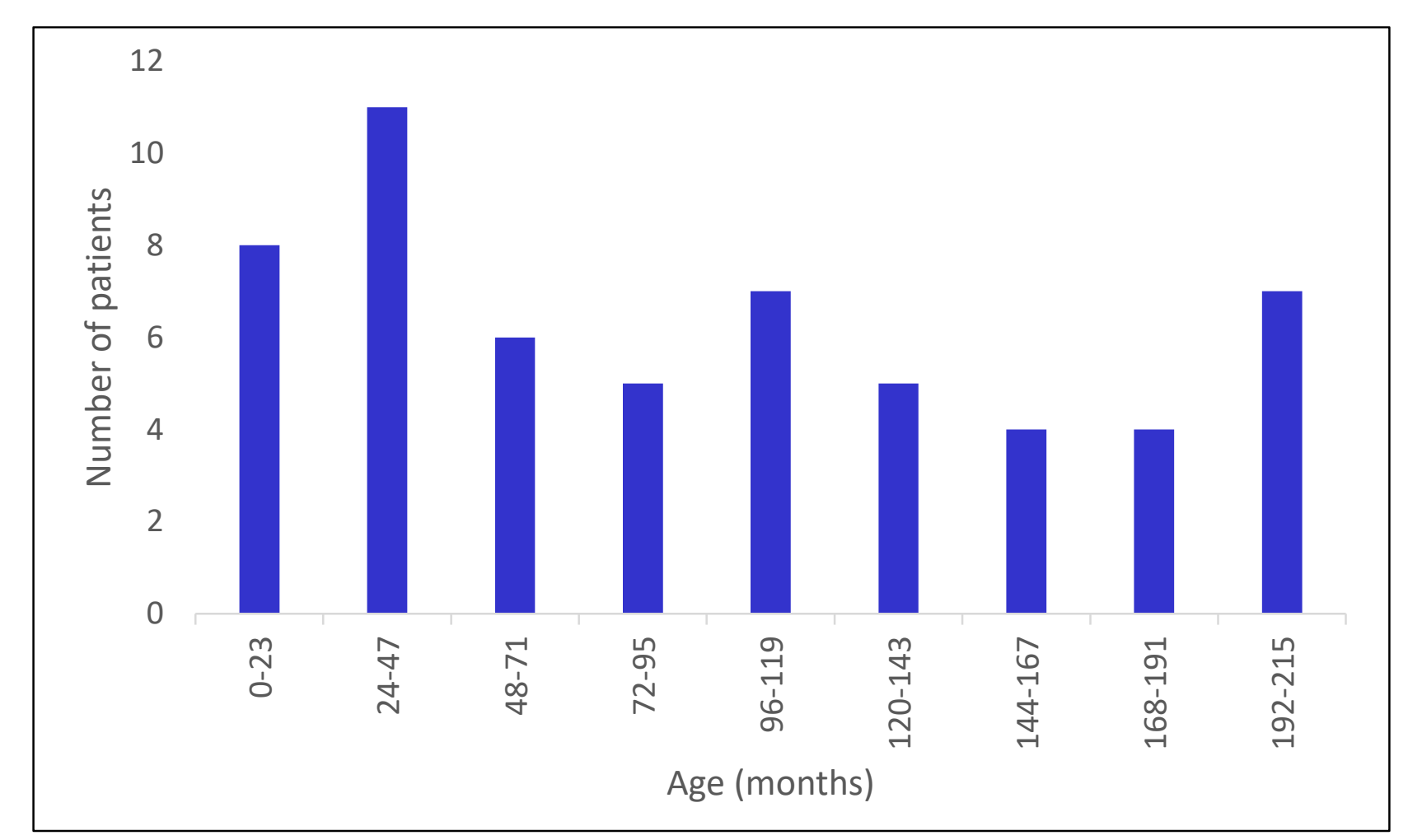

Results: We treated 29 male and 29 female patients, median age 90 months (IQR 32.5-144 months). Half of our patients had anamnestic antecendent infection (respiratory illness in $72.41 \%$ ). Most of patients presented during winter (34.48\%) and spring (31.03\%). Median of disease duration before admission was 4 days (IQR 2-8 days). Fever was present in $72.41 \%$ of patients. 23 patients had seizures during disease course. At admission, GCS was 15 in $66.67 \%, 14$ in $15.69 \%, 10-12$ in $9.8 \%$ and $<10$ in $7.84 \%$ of patients. Our patients had median (mononuclear) pleocytosis of 130 (IQR 23-281). Initial MRI was abnormal in 87.93\% of patients. Predominantely affected regions were infratentorial region (37.25\%), subcortical region (35.29\%), deep gray matter - thalamus and basal ganglia (35.29\%), periventricular area (33.33\%), cortical (29.41\%) and jucstacortical area (19.61\%). 21.57\% of patients had more than six lesions. Treatment regimens were as following: pulse doses of steroids in $64.29 \%$, steroids+plasmapheresis in 12.5\%, steroids+plasmapheresis+IVIG in $12.5 \%$. At hospital discharge, 24 (41.38\%) patients had sequele - most common was lower limb weakness (25\%).

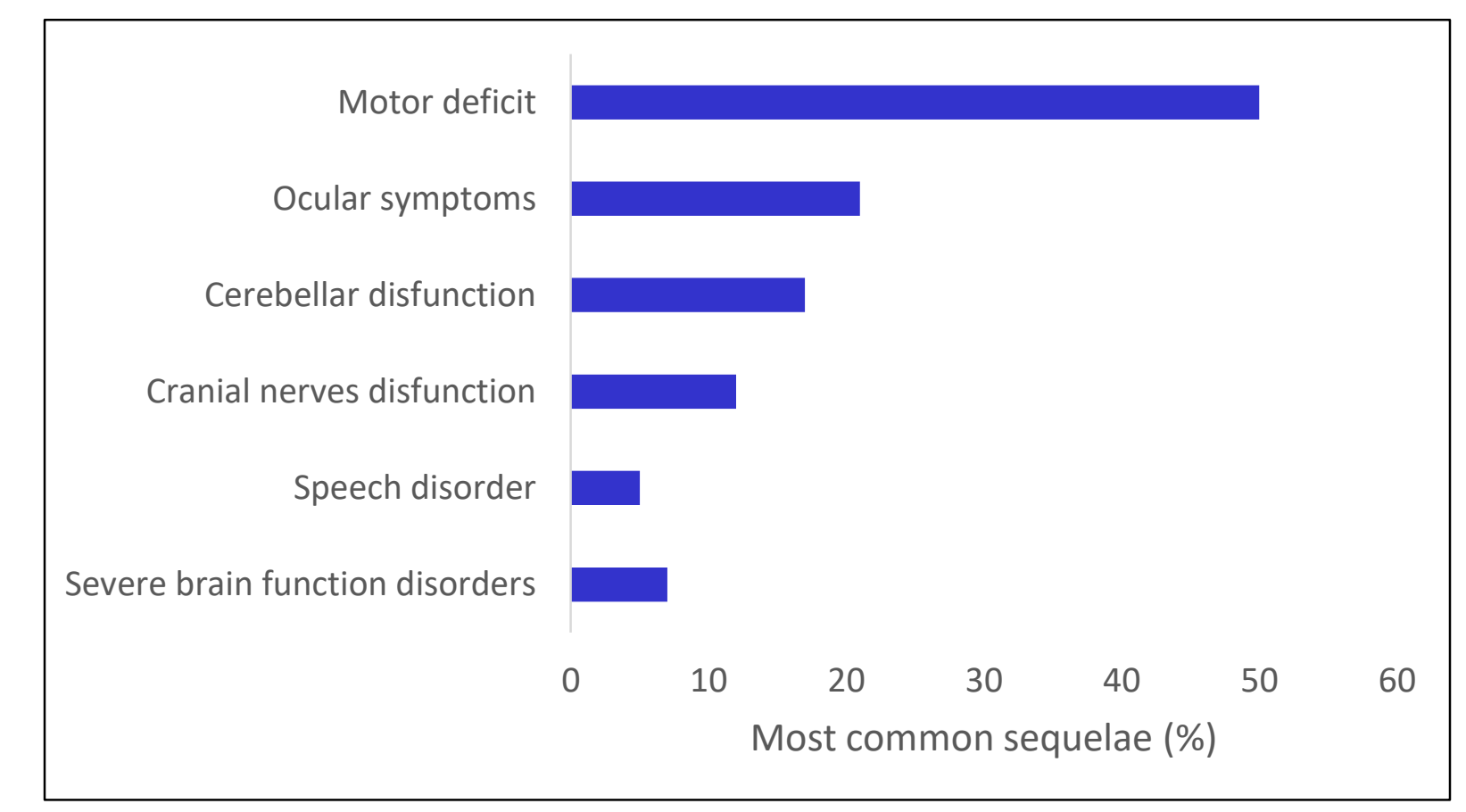

*At hospital discharge

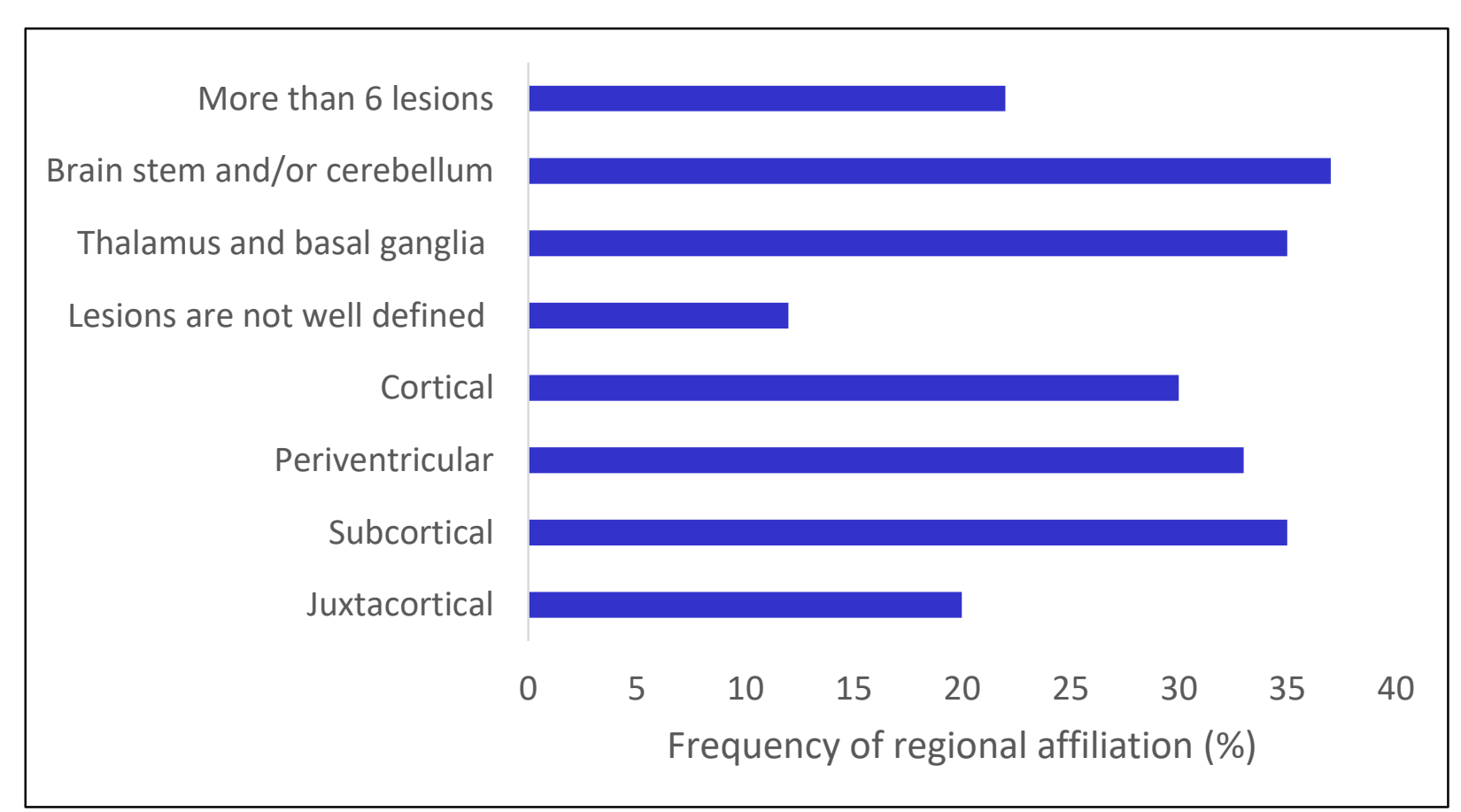

${ }^{*}$ MRI findings localisation

Conclusions: Patient age, history of antecedent infection, winter and spring seasonal peaks in disease presentation, clinical findings (fever, seizures, encephalopathy), laboratory findings (lymphocytic pleocytosis) and suggestive MRI findings are some of the findings commonly associated with this disease. 